\title{
La morphologie des tissus urbains et périurbains à travers une lecture fractale
}

The morphology of urban and peri-urban fabric, based on a fractal analysis Grundrissmuster der städtischen und randstädtischen Flächen in fraktaler Geometrie

\section{P. Frankhauser}

\section{(2) OpenEdition}

\section{Journals}

\section{Édition électronique}

URL : http://journals.openedition.org/rge/268

DOI : $10.4000 /$ rge.268

ISSN : 2108-6478

Éditeur

Association des géographes de l'Est

Édition imprimée

Date de publication : 1 juin 2005

Pagination : 145-160

ISSN : 0035-3213

\section{Référence électronique}

P. Frankhauser, «La morphologie des tissus urbains et périurbains à travers une lecture fractale»,

Revue Géographique de l'Est [En ligne], vol. 45 / 3-4 | 2005, mis en ligne le 10 juin 2009, consulté le 21 décembre 2020. URL : http://journals.openedition.org/rge/268 ; DOI : https://doi.org/10.4000/rge.268

Ce document a été généré automatiquement le 21 décembre 2020.

Tous droits réservés 


\section{La morphologie des tissus urbains et périurbains à travers une lecture fractale ${ }^{1}$}

The morphology of urban and peri-urban fabric, based on a fractal analysis Grundrissmuster der städtischen und randstädtischen Flächen in fraktaler Geometrie

P. Frankhauser

Nous remercions le PUCA (Plan Urbanisme, Construction, Architecture) pour le financement des recherches récentes et le développement du logiciel fractalyse, ainsi que tous les participants à ce projet de recherche. Plus particulièrement, nous remercions Gilles Vuidel pour le développement du logiciel fractalyse et les institutions qui nous ont mis à disposition les données cartographiques.

\section{Introduction}

\section{A. Quelle référence pour maîtriser l'étalement urbain ?}

1 L'étalement urbain et la maîtrise de ses conséquences restent un défi important de l'aménagement du territoire. Les conséquences de l'étalement urbain, la consommation d'espace, la génération de trafic et ses impacts environnementaux sont de bonnes raisons pour mettre en cause cette évolution. Mais les concepts traditionnels d'une ville compacte sont-ils adaptés pour trouver des solutions réalistes? Comme l'ont constaté beaucoup d'observations les succès des opérations visant à limiter l'étalement urbain sont limités et les tentatives de densification sont souvent mal accueillies par la population concernée (Frank (1987), Remy (1994), Fouchier (1995)).

2 Les références traditionnelles ne permettent apparemment pas de trouver des réponses appropriées à ce phénomène. On peut donc poser la question de la nécessité de trouver d'autres approches pour mieux comprendre la morphologie de ces tissus souvent 
perçus comme "amorphes", donc "sans forme ». Mieux comprendre veut aussi dire mieux prendre en compte les raisons socio-économiques de cette évolution. À partir de cette connaissance on peut imaginer de développer des concepts différents pour trouver des réponses réalistes au défi de l'étalement urbain.

\section{B. Quelques observations sur la morphologie des tissus urbains}

3 Les tissus urbains des zones urbaines actuelles montrent certaines caractéristiques qu'on peut associer à un principe d'emboitement d'échelles qui apparaît dans la forme même des taches urbaines : comme le montre l'exemple de la figure 1 leurs bordures présentent de grandes "baies ", qui pénètrent dans la surface bâtie, et qui sont souvent elles-mêmes composées d'un nombre croissant de plus petites baies, ce qui donne au final l'aspect tentaculaire des agglomérations. Si l'on met en relation la longueur de la bordure urbaine et la surface bâtie intra-urbaine, on s'aperçoit qu'il existe une relation forte entre les deux variables, mais celle-ci est en contradiction avec la géométrie euclidienne: la valeur mesurée pour la surface est proportionnelle à celle du périmètre, alors qu'elle devrait être proportionnelle au carré de celui-ci dans le cas de la géométrie euclidienne (Frankhauser (1994)).

4 La figure 1 montre aussi que les agglomérations contemporaines sont composées d'un grand nombre d'agrégats bâtis, dont la taille est variée et suit souvent une logique d'emboîtement d'échelles : villages ruraux et périurbains, bourgs-centres, ville-centre... Une logique similaire existe pour les vides (i.e. les lacunes) à l'intérieur des tissus urbains : il existe peu de très grandes places vides, un nombre plus élevé d'espaces libres de taille moyenne et enfin un grand nombre de petits espaces libres (cours intérieures par exemple). Dans beaucoup de cas l'habitat n'est pas réparti de manière uniforme, mais se concentre le long d'axes de transport. Il existe ainsi des zones dans lesquelles le bâti est beaucoup plus concentré qu'ailleurs, ce qui se traduit par une baisse globale de la densité en s'éloignant des centres densément peuplés.

5 Comme le montre la figure $1 \mathrm{~b}$, on retrouve certaines de ces caractéristiques si on se situe à une échelle intra-urbaine. Comme à l'échelle de l'agglomération la surface n'est pas répartie de façon uniforme : les bâtiments forment des agrégats laissant vides des espaces de taille très différentes: des poches non bâties pénètrent dans les zones construites et séparent les bâtiments ou, dans les zones plus densifiées les îlots urbains.

6 La forme de ces tissus urbains doit être considérée comme une expression de notre mode de vie actuel. Les zones résidentielles implantées dans un environnement rural paraissent garantir une vie calme, à l'abri de l'agitation qui caractérise les centres denses. Comme par ailleurs, dans notre société, on se déplace facilement, le fait de vivre «à la campagne » ne veut plus dire qu'on se coupe des aménités qu'offrent les centres urbains. Il est possible d'accéder sans trop de contraintes non seulement au lieu de travail, mais aussi aux endroits où l'on trouve une offre diversifiée de loisirs et de services. Ainsi, les nouveaux espaces urbains ne sont pas le résultat d'une politique d'aménagement cohérente, mais sont plutôt issus de multiples interactions entre acteurs publics et privés. Le processus d'urbanisation doit de ce fait être considéré comme un processus d'auto-organisation, ce qui transparaît dans la désignation de «ville émergente» en France (Dubois-Taine, Chalas (1997)) ou dans les termes de Zwischenstadt (« l'entre-ville ») ou de Verstädterungslandschaft ("paysage d'urbanisation ») en Allemagne (Humpert et al. (1991), Sieverts (1997)). 
7 Si le fonctionnement des espaces urbains paraît aujourd'hui plus clair, la question des références servant à étudier et à réfléchir à leur organisation spatiale persiste: comment décrire la forme des "taches d'huile» observées? Quelles sont leurs caractéristiques? Peut-on en tirer des conclusions pour orienter le futur développement des espaces urbains vers une solution qui intègre à la fois, les aspirations des résidents et les objectifs d'un développement?

Figure 1 : (a) Une représentation simplifiée de la zone métropolitaine de Stuttgart. On identifie les caractéristiques morphologiques décrites dans le texte. (b) Une représentation détaillée de la surface bâtie d'une commune périphérique.

Image 1

\section{L'approche fractale des tissus urbains}

\section{A. Vers d'autres modèles pour caractériser la forme des tissus urbains}

8 La présence d'un principe d'emboîtement d'échelle dans les tissus urbains a incité différentes équipes de recherche à recourir à une approche géométrique alternative, la géométrie fractale, qui est par définition, multi-échelle ${ }^{2}$. En utilisant des méthodes d'analyse basées sur cette approche, on peut mettre en évidence l'existence d'un certain type d'organisation spatiale dans les tissus urbains que l'on ne retrouve pas en recourant à d'autres approches, par exemple en se référant à la densité. En introduisant des descripteurs morphologiques fractals, la comparaison et la classification des tissus urbains deviennent dès lors possible. Dans un premier temps, ces investigations ont porté sur de vastes zones métropolitaines analysées à partir de représentations cartographiques simplifiées (par exemple, $1: 500.000)$ (Batty et Longley (1986), (1994), Batty et Kim (1992), White et Engelen (1994), Frankhauser (1988), (1994)). Ces travaux ont été approfondis et en partie appliqués à l'échelle des quartiers en utilisant des bases de données cartographiques plus détaillées (Batty et Xie (1996), Frankhauser (1997), François, N. et al. (1995)), Frankhauser et Genre-Grandpierre (1998), Frankhauser, (2000), Benguigui et al. (2000), Shen (2002), Schweitzer and Steinbrick (2002), De Keersmaecker et al. (2003), (2004), Frankhauser (2003), Frankhauser et Tannier (2005)).

9 Au-delà de l'analyse morphologique il est possible de concevoir des modèles de références qui peuvent servir à illustrer les propriétés principales de différents types de formes urbaines. Des réflexions conceptuelles permettent de répondre à des objectifs d'aménagement urbain visant à limiter la consommation d'espace en assurant une bonne qualité de vie.

10 Afin d'illustrer les particularités de la géométrie fractale, nous avons choisi de présenter ici plusieurs modèles fractals théoriques, qui transcrivent des propriétés caractéristiques des tissus urbains. Par définition, ces fractales sont construites selon un principe d'emboîtement d'échelle : en réitérant une règle de construction, on génère une structure fortement hiérarchisée qui paraît de plus en plus complexe. 


\section{Deux modèles de base : la poussière de Fournier et le tapis de Sierpinski}

11 Comme nous l'avons vu, à l'échelle des îlots urbains, la surface bâtie est constituée de bâtiments isolées ou de groupes de bâtiments, donc d'éléments détachés qui forment des agrégats. Il est possible d'illustrer ce type de structure spatiale à travers un certain type de fractal, les poussières de Fournier. La figure 2 montre de quelle manière une telle fractale peut être construite.

Figure 2 : La construction d'une poussière de Fournier par itération. Les éléments du générateur sont isolés.

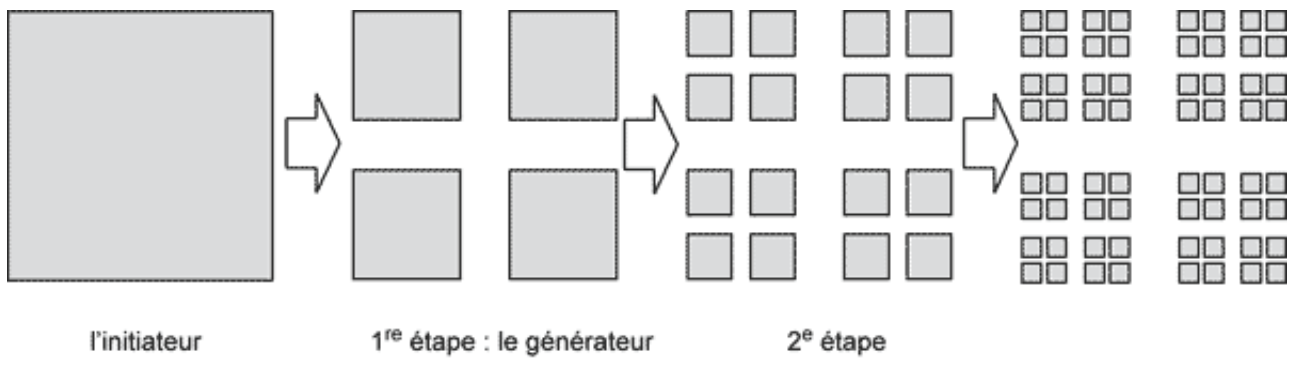

12 L'initiateur, un carré, est remplacé, lors d'une première étape, par $N=4$ répliques dont la longueur de base est réduite par un facteur $r=2 / 5$.Cette règle de construction est appelée générateur. On l'applique, lors de l'étape suivante, à chacun des carrés générés précédemment. La figure 2 montre qu'il est possible de poursuivre cette itération. Nous désignons désormais les carrés qui constituent la fractale à une étape d'itération donnée comme " éléments ", et l'ensemble de ces éléments forme la " surface occupée » que nous associons à la surface bâtie en comparant la fractale à un tissu urbain. En poursuivant l'itération, la surface totale des carrés générés tend vers zéro. Nous observons que l'itération génère des agrégats qui sont séparés par des bandes vides de tailles différentes. Ces bandes forment un système hiérarchique qui nous rappelle celui de la voirie avec peu d'avenues larges et un nombre croissant de rues de plus en plus étroites. Il est possible de montrer (Frankhauser (1994)) que cette hiérarchie suit un principe particulier, la distribution de Pareto-Zipf, connue en géographie et en économie par exemple pour décrire la loi rang-taille des villes, qui représente la distribution de la population dans un système de villes (Pumain (1981)).

Soulignons que les propriétés fondamentales d'une fractale ne dépendent ni de la position des éléments dans le générateur, ni de la forme de la figure initiale. Le seul aspect qui entre en jeu est le principe d'emboîtement d'échelle qui est déterminé par le nombre $N$ et le facteur $r$.

En considérant la représentation graphique simplifiée de la figure 1a nous avons découvert une autre caractéristique des tissus urbains. A cette échelle les villes ressemblent plutôt à des taches dont les bordures sont très tortueuses. Cet aspect peut être représenté par un autre modèle fractal, similaire au premier, le tapis de Sierpinski. Nous avons représenté sur la figure 3 la procédure qui permet de construire une telle fractale.

15 Le principe reste le même sauf que dans le générateur les éléments se touchent maintenant. Ainsi la fractale est constituée d'un seul agrégat à travers les étapes. Cependant, comme on le remarque, la bordure devient de plus en plus tortueuse. 
Figure 3 : Les trois premières étapes de construction d'un tapis de Sierpinski ((a) (b) (c)) et le sytème d'emboîtement d'échelles des parties vides, illustré pour la troisième étape (d).

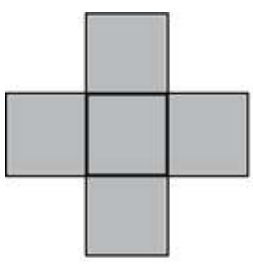

(a)

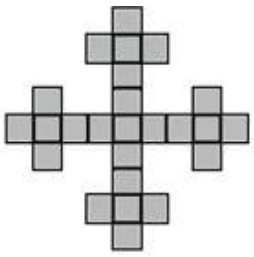

(b)

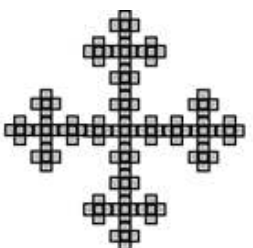

(c)

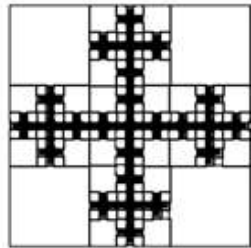

(d)

Nous pouvons considérer cette figure comme un modèle simplifié d'une ville qui s'est étalée le long de deux axes de transport perpendiculaires que nous désignons comme axes de "premier ordre » du réseau. Cette forme de croix apparaît dans la première étape d'itération, qui peut être interprétée comme une représentation cartographique très grossière de la ville. En passant à des représentations plus fines, on découvre progressivement de plus en plus de détails de ce tissu urbain. Ainsi la figure (b) fait apparaître une ville qui s'est non seulement étalée le long des axes principaux, mais aussi le long d'axes de "second ordre " qui se greffent sur les axes principaux. La troisième figure (c) montre qu'il existe aussi des axes de troisième ordre, etc. Apparemment, une telle ville s'est développée à proximité des « carrefours » du réseau de transport sous-jacent. Ainsi, le tissu urbain garde, en dépit d'une bordure complexe, une certaine compacité. Celle-ci s'exprime par l'existence de quatre zones carrées nonurbanisées de grande taille (figure 3d). A une échelle plus fine, on observe 20 zones vides de taille moyenne et 100 zones de petite taille. Comme pour les bandes nonoccupées dans la poussière de Fournier la répartition de ces zones vides rend donc compte du principe sous-jacent d'emboîtement d'échelles. Ceci devient encore plus évident en considérant le tapis de Sierpinski de la figure 4a. Dans ce cas, la bordure extérieure de la fractale est lisse ; l'allongement de la bordure apparaît si l'on considère l'ensemble des bordures de toutes les lacunes situées à l'intérieur qui sont générées progressivement lors des étapes d'itération.

Une « ville fractale » comme celle de la figure 3 offre un certain nombre d'avantages en terme d'accessibilité à divers types "d'aménités». Ceci devient évident si nous comparons ce modèle à deux autres modèles pour lesquels nous avons conservé la surface bâtie, mais où nous l'avons répartie de façon différente (Frankhauser et al. (1998)) :

-(1) une ville linéaire dont la largeur est celle des carrés de notre ville fractale ;

- (2) une ville compacte de forme quadratique (figure $4 \mathrm{~b}$ ).

Dans les trois modèles, nous avons localisé un certain nombre de centres de service (centre de commerce, services publics...). Dans le cas de la structure fractale, nous avons supposé que ceux-ci sont localisés sur les intersections des différentes branches et qu'il existe une hiérarchie stricte des services offerts : le centre ancien au cœur de l'agglomération offre tous les services de haut de gamme, mais aussi tous les services des niveaux inférieurs. Les intersections générées lors de la première étape d'itération sont des centres secondaires d'ordre 1 qui réunissent tous les services exceptés les niveaux les plus élevés, etc. (figure 4c). Nous avons gardé le même nombre de centres de chaque niveau hiérarchique pour les deux autres modèles. Dans le cas de la ville compacte nous avons choisi une répartition homogène des centres de service et une disposition des centres secondaires qui optimise leur accessibilité (figure 4b), tandis 
que pour la ville linéaire, il a été possible de concevoir une disposition parfaitement symétrique et homogène.

Figure 4 : (a) Un tapis de Sierpinski assez compacte en deuxième étape d'itération.

(b) Un modèle d'une ville compacte dans laquelle on a répartie des centres de service de différents niveau : au centre, en noir le centre principal, en gris dégradé les centre de second, troisième et quatrième ordre (cf. texte).

(c) Partie d'une « ville fractale » dans laquelle les centres de services sont localisés aux intersections des axes (cf. texte). La structure complète est constituée de cinq de ces parties placées sous forme d'une croix.

(d) Partie d'une "ville linéaire ", composée de 125 éléments. Chaque cinquième élément est un centre de service du niveau le plus bas. Cependant le centre de service situé au milieu de cinq centres offre un niveau de service supérieur. Le niveau hiérarchique suivant se trouve au milieu de 25 sites. Enfin le centre principal se situe au cœur de la structure.

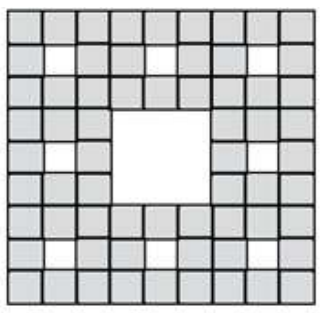

(a)

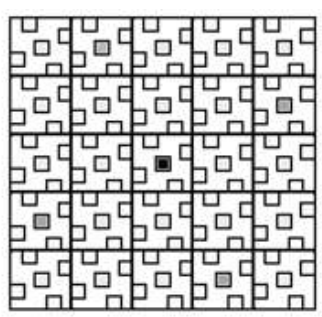

(b)

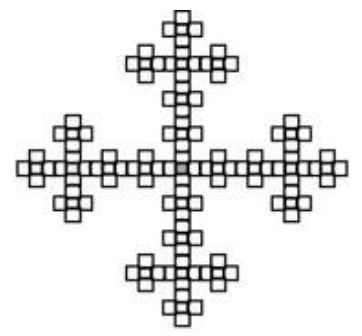

(c)

(d)

Pour chacune de ces villes-modèles, nous avons déterminé les distances moyennes aux centres de services les plus proches, considérant chaque niveau hiérarchique. Nous avons aussi calculé la distance minimale pour accéder à la bordure des villes, c'est-àdire l'accessibilité aux zones non-bâties " rurales ", que nous avons considérées comme zones récréatives. Les résultats obtenus montrent que la «ville fractale » se situe entre les deux autres modèles urbains en ce qui concerne les distances aux centres de service et aux zones récréatives: la distance moyenne au centre principal est 2,21 fois plus élevée que pour la structure compacte, mais pour les centres de service de troisième ordre, autrement dit, les centres de proximité, les distances sont équivalentes entre la ville fractale et la structure compacte. En comparaison avec la «ville linéaire », la ville fractale est évidemment largement avantagée. Par exemple, la distance moyenne au centre de service principal est $18 \%$ de celle observée pour la ville linéaire. Concernant maintenant la proximité aux zones vertes, la ville fractale se trouve en revanche plus proche de la structure linéaire. Ainsi, pour atteindre la zone verte, la distance moyenne est 16,5 fois plus élevée sur le réseau compact que sur la fractale, mais elle est seulement légèrement plus élevée sur la fractale que sur le réseau linéaire.

On pourrait donc en conclure qu'une "ville fractale» est intéressante pour des individus fréquentant moins les centres hiérarchiques les plus élevés (centre principal et sous-centre de premier ordre) que les sous-centres de proximité, tout en recherchant la proximité aux zones vertes récréatives.

21 Les exemples d'objets fractals, notamment ceux des figure 2, 3d et 4a, montrent que la propriété principale des structures fractales est l'existence d'un principe d'emboîtement d'échelles qui s'exprime notamment par l'existence de zones vides de tailles différentes. Ainsi les éléments de la fractale sont répartis de façon inégale, il existe des zones de forte et de faible concentration de la surface occupée : une fractale 
n'est ni dense, ni diluée. Comme la densité est uniquement constante dans le cas où les éléments constitutifs d'une structure, par exemple les bâtiments dans un tissu urbain, sont distribués de façon uniforme sur la surface, la densité est soumise à de fortes variations locales dans une fractale.

\section{Des modèles mixtes}

Il est possible de s'approcher encore plus des aspects morphologiques dont nous avons fait allusion en combinant les deux logiques, celle du tapis de Sierpinski et celle de la poussière de Fournier. Ainsi la fractale de la figure $5 \mathrm{a}$ est constituée d'un agrégat principal entouré d'agrégats de taille différente. On pourrait identifier l'agrégat central à une grande ville qui est entourée de sous-centres situés à une certaine distance de celle-ci. Dans la proximité de chaque ville se trouve un certain nombre de petites villes.

La figure $5 b$ pourrait plutôt être associée à une structure intra-urbaine composée d'îlots urbains.

Figure 5 : Deux fractales mixtes en deuxième étape d'itération : l'exemple (a) ressemble aux semis d'habitats et (b) aux tissus intra-urbains.

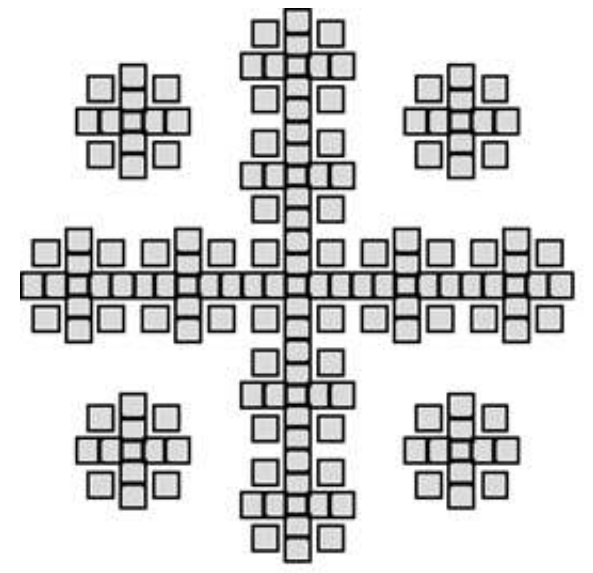

(a)

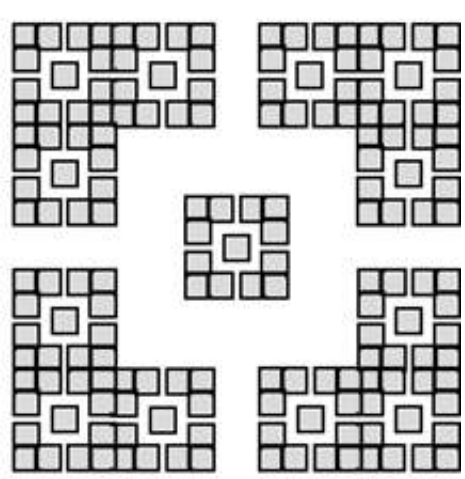

(b)

\section{Un modèle spécifique pour la bordure urbaine : le téragone}

Une des caractéristiques importantes de l'étalement urbain est l'aspect tortueux de la bordure d'une agglomération. Le fait que la bordure du tapis de Sierpinski s'allonge au cours des itérations est un indice montrant que les fractales peuvent servir à étudier ce phénomène. Ceci devient encore plus évident si l'on recourt à un autre modèle, celui du téragone. Ici, on construit un objet de topologie linéaire dont le générateur est présenté par la figure 6. La figure géométrique initiale est une section de droite de longueur donnée. Le générateur remplace cette figure par un polygone composé de huit sections de droite de longueurd'un quart. Cette logique est ensuite appliquée à chacune des huit sections de droite, etc. En poursuivant l'itération, la longueur de cet objet tend vers l'infini. Dans la figure $6 \mathrm{~b}$, quatre de ces courbes sont disposées bout à bout. Elles délimitent ainsi une surface qui est un carré au départ et dont la superficie demeure constante au cours des itérations. Cette structure est appelée "téragone». En considérant cette structure comme représentant une ville, nous supposons que la 
masse bâtie est répartie de façon homogène à l'intérieur de la ville. Nous pouvons donc interpréter le carré initial de la figure comme le modèle d'une ville de surface donnée dont le tissu bâti est très homogène et qui est délimité par une enceinte quadratique. $\mathrm{Au}$ cours de l'itération, la structure s'étend progressivement en surface, la bordure ressemblant, par son aspect sinueux, de plus en plus aux tissus urbains réels.

Figure 6 : Construction progressive d'un téragone dont la surface totale reste constante au fil des itérations. La répartition de la surface noire est uniforme à l'intérieur, mais la bordure est fractale : sa longueur tend vers l'infini.
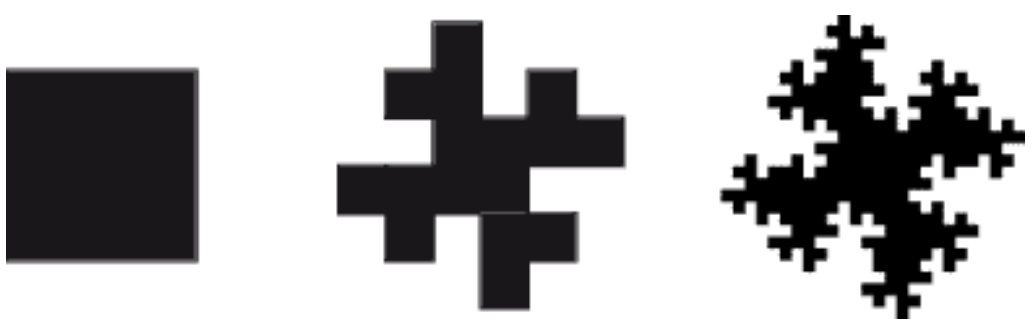

Considérons maintenant les étapes d'itération comme différents modèles d'une ville. La figure initiale, la "ville carrée", offre peu de terrains situés en bordure de la ville (proche de la campagne). L'allongement progressif de la bordure lors des prochaines étapes permet à un nombre croissant d'acheteurs d'acquérir des terrains situés en bordure de la ville. Ceci leur permet de bénéficier de la proximité à un paysage ouvert tant que les poches vertes qui entrent dans la « surface bâtie » ne sont pas trop petites. Il est possible de montrer qu'en passant de la figure initiale aux étapes d'itération suivantes, le nombre de terrains situés en bordure de la ville double à chaque fois. En outre, chaque étape génère des poches non-occupées, donc "vertes ", qui pénètrent de plus en plus profondément dans la surface bâtie. On vérifie également que la distance minimale entre la bordure et le centre de la structure tend vers une limite qui est égale au tiers de la taille du carré initial. De même, la taille de la structure générée ne dépasse pour aucune étape d'itération $5 / 3$ de cette longueur. Celle-ci correspond à la distance maximale qui existe entre la bordure et le centre. Par ailleurs, comme la surface bâtie à l'intérieur de la structure reste identique pour toutes les itérations, il n'y a aucune différence de consommation d'espace entre les différentes étapes considérées.

On pourrait imaginer qu'une telle structure étalée diminue l'accessibilité au centre de la ville. Nous avons donc calculé le gain d'accessibilité à la bordure en calculant pour les premières étapes d'itération la distance minimale moyenne à la bordure. D'autre part, nous avons calculé la distance minimale au centre. On constate pour la première étape d'itération un allongement de $12 \%$ de la distance moyenne au centre, donc une perte d'accessibilité. En revanche, la distance à la bordure tombe à $56 \%$ de sa longueur initiale. Pour la deuxième étape d'itération, les modifications sont moins spectaculaires : la perte pour accéder au centre est de $4 \%$ contre un gain de $9 \%$ pour atteindre les bordures ${ }^{3}$. Ainsi le gain d'accessibilité aux zones vertes, que nous supposons entourer la zone bâtie, est plus important que la perte d'accessibilité au centre ville (Frankhauser (2000)).

Ceci montre les avantages non-négligeables d'une structure étalée - sous conditions qu'elle soit organisée de manière « rationnelle ». 


\section{B. Comment mesurer la fractalité des tissus urbains}

L'introduction d'indicateurs qui quantifient l'organisation multi-échelle d'une structure permet de mettre en évidence l'existence de principes d'ordre que les mesures habituelles ne permettent pas de découvrir. Il est ainsi possible d'identifier des changements dans l'organisation spatiale des villes à certaines échelles. En comparant différents tissus urbains, il est également envisageable d'introduire des classes morphologiques de tissus et de vérifier si les classes identifiées correspondent à des situations urbanistiques particulières.

Sur le plan mathématique, en raison de la règle stricte utilisée pour construire une fractale théorique, le principe d'emboîtement d'échelle suit une loi bien définie. Celle-ci traduit le fait qu'au cours de l'itération, un nombre $N(\varepsilon)$ croissant d'éléments est généré, dont la taille $\varepsilon$ est de plus en plus petite. Cette loi peut être écrite sous la forme ${ }^{4}$ :

$$
N(\varepsilon) \sim \varepsilon^{-D}
$$

Selon cette loi, le paramètre $\mathrm{D}$ représente la dimension fractale. Pour des fractales construites $\mathrm{D}$ est directement lié aux nombres d'éléments $\mathrm{N}$ et au facteur de réduction $\mathrm{r}$ qui caractérisent le générateur :

$$
\mathrm{D}=-\frac{\log N}{\log r}
$$

En insérant les paramètres $N$ et $r$ on obtient pour la poussière de Fournier de la figure 3 une dimension $D=1,51$ et pour le tapis de Sierpinski de la figure $4 D=1,49$.

Il est possible d'appliquer la définition de la dimension fractale à un objet géométrique euclidien. On obtient alors pour une surface sur laquelle on a réparti une masse bâtie de façon uniforme la valeur $D=2$ et pour un point isolé la valeur $D=0$.

Pour un tissu urbain, la valeur de la dimension fractale caractérise le degré de concentration de la masse bâtie à travers les échelles dans une zone choisie de la structure, autrement dit, le degré de non-uniformité de la répartition de cette masse $\mathrm{e}^{5}$. Ainsi une valeur proche de deux correspond à une structure assez uniforme, donc faiblement hiérarchisée, tandis qu'une valeur proche de zéro caractérise une structure fortement hiérarchisée, présentant des concentrations de masse importantes à quelques endroits.

Ce concept de mesure s'applique également à des objets de topologie linéaire, comme la bordure d'une poussière de Fournier, d'un tapis de Sierpinski ou d'un téragone. Tandis que la dimension d'une ligne droite est toujours $D=1$, celle d'une bordure fractale est supérieure à la valeur $D=1$.Dans le cas du tapis de Sierpinski, la dimension de la bordure $D_{\text {bord }}$ et celle de la surface $D_{\text {surf }}$ sont identiques: en effet, si le nombre d'itérations tend vers l'infini, la bordure et la surface occupée se confondent. Un tapis de Sierpinski se situe ainsi, par sa morphologie, entre une structure linéaire et une surface uniformément occupée. Pour le téragone, la situation est différente : la surface 
est uniforme et donc de dimension $D_{\text {surf }}=2$, en revanche la dimension de la bordure est $D_{\text {bord }}>1$.

Pour les fractales mixtes telles que celle de la figure 5a, il est possible d'introduire deux types de dimensions :

- une dimension fractale qui caractérise la forme de chaque agrégat indépendamment de la disposition des agrégats les uns par rapport aux autres. Nous utilisons cette possibilité pour introduireune dimension $D_{\text {bord/agr }}$ qui décrit la forme de l'agrégat principal de l'agglomération et notamment la tortuosité de sa bordure ;

- une seconde dimension fractale qui décrit la répartition de l'ensemble de tous les agrégats. Cette approche nous servira à définir une dimension $D_{\text {bord/tot }}$ qui caractérise l'ensemble des bordures urbaines. Une valeur basse indique que les agrégats sont fortement hiérarchisés, tandis qu'une valeur proche de deux montre une tendance vers une distribution uniforme des bordures.

En s'appuyant sur ces différents types de dimensions nous avons testé deux indices ( $c f$. aussi Frankhauser (2003)) :

- un indice de fragmentation

$$
\varphi=\frac{D_{\text {bord/tot }}}{D_{\text {bord/agr }}}-1
$$

dont la valeur varie entre zéro et un. La valeur zéro indique que la structure est constituée d'un seul agrégat tandis qu'une valeur proche de un caractérise un tissu fragmenté constitué d'un grand nombre d'îlots qui couvrent la surface de façon quasi uniforme ;

- un indice de dendricité

$$
\delta=2-\frac{D_{\text {surf }}}{D_{\text {bord/agr }}}
$$

qui se situe dans la même fourchette de valeur. Une structure compacte et lisse coïncide avec la valeur zéro et une valeur proche de un correspond à un tapis de Sierpinski pour lequel les dimensions de la bordure et de la surface se confondent (cf. figure 2).

Différentes méthodes ont été développées afin d'étudier le comportement fractal d'une structure, même si celle-ci n'est pas issue d'une construction itérative. De façon générale on peut distinguer trois types de méthodes d'analyse fractales qui sont utilisés dans l'analyse des tissus urbains :

\section{Les méthodes de comptage}

\subsection{L'analyse radiale}

On détermine la masse bâtie, c'est-à-dire pour une carte numérisée, le nombre de pixel occupés $\mathrm{N}(\varepsilon)$ qui se situe à l'intérieur d'un cercle de rayon $\varepsilon$ qui entoure un point de comptage choisi. Celui-ci peut être situé au cœur du centre historique, mais aussi en périphérie. En faisant varier le rayon e on obtient une information sur la baisse radiale de la densité dans la proximité du point de comptage. La fonction $\mathrm{N}(\varepsilon)$ montre souvent des irrégularités caractéristiques pour certaines distances $\varepsilon_{\text {crit }}$ qui permettent de localiser facilement des ruptures morphologiques dans un tissu urbain (figure 6). 


\subsection{L'analyse de corrélation} échelle des quartiers et l'autre, à l'échelle de la ville dans son ensemble. Un mode de représentation spécifique des courbes d'analyse s'avère dans ce cas utile: en partant d'une représentation bi-logarithmique des courbes d'analyse $N(\varepsilon)$, on détermine pour celle-ci la pente $\alpha$ entre chaque point et son point voisin. On obtient ainsi une série de valeurs $\alpha(\varepsilon)$ que l'on peut représenter en fonction de la distance $\varepsilon$ à laquelle elle correspond. Nous avons désigné ces représentations par l'expression «courbes du comportement scalant ». Pour une fractale théorique, $\alpha(\varepsilon)$ doit être constant et égale à la dimension fractale : la courbe du comportement scalant a alors la forme d'une droite horizontale. Dès lors pour une ville, il est facile d'identifier les valeurs $\varepsilon_{\text {crit }}$ pour lesquelles le comportement scalant change, ce qui indique un changement dans l'organisation spatiale du tissu à l'échelle qui correspond à la valeur $\varepsilon_{\text {crit }}$. La figure 6 montre un exemple d'une courbe du comportement scalant issue d'une analyse radiale.

\section{Une approche comparative de villes européennes}

Comme nous avons pu le constater, la morphologie des tissus urbains présente des caractéristiques qui rappellent le principe d'emboîtement d'échelles des objets fractals. Il n'est donc pas surprenant que l'analyse fractale se soit en effet avérée un instrument pertinent pour étudier la répartition du bâti dans les villes. Les divers travaux entrepris montrent que les analyses fractales permettent de distinguer soit, à travers les valeurs des dimensions fractales soit, par l'aspect des courbes empiriques obtenues, différents types de quartiers dans les tissus urbains. Il est ainsi possible d'étudier le lien entre le contexte d'urbanisation et la morphologie urbaine. En effet, on peut supposer que le 
tissu d'une agglomération dont l'évolution a été fortement marquée par l'industrialisation est différent d'une ville de caractère plutôt commercial ou administratif. La réglementation nationale et les orientations de la politique locale sont également des facteurs susceptibles d'influencer l'organisation spatiale d'une ville, tout comme sa position topographique.

Étudier l'influence de ces facteurs sur la morphologie des tissus urbains était un des objectifs d'un programme de recherche, récemment financé par le PUCA. Nous nous référons dans cet article essentiellement aux résultats obtenus dans le cadre de ce projet de recherche ${ }^{6}$ (Frankhauser (2003)). Deux niveaux d'analyse ont été considérés : celui de l'agglomération et celui des quartiers. Le choix des quartiers a été fait dans l'objectif de chercher à associer des valeurs de dimensions fractales à différents types de quartiers (quartiers pavillonnaires, grands ensembles, centres anciens...). Il est évident que chacun de ces quartiers correspond au contexte politique ou urbanistique particulier dans lequel il a été construit. A l'échelle des agglomérations, l'intérêt était davantage l'analyse de la forme des "tache d'huile ", donc, de leur bordure. Pour réaliser les analyses, le logiciel fractalyse ${ }^{7}$ a été développé. Il permet d'étudier des zones urbaines étendues, tout en gardant une information détaillée sur les tissus bâtis.

\section{A. L'organisation spatiale à l'échelle des agglomérations}

Nous commençons par la présentation de quelques résultats obtenus à une échelle agrégée des agglomérations. Ces résultats se réfèrent à des représentations graphiques simplifiées comme celle de la figure 1. Nous présentons les résultats obtenus pour trois zones métropolitaines dont la taille et le contexte d'urbanisation sont très différentes : Berlin, Stuttgart et Londres.

Dans chaque cas nous avons déterminé les dimensions fractales de la surface bâtie $D_{\text {surf }}$ Pour étudier la forme de la bordure urbaine, nous avons mesuré :

- d'une part, la dimension fractale de la bordure de l'ensemble des agrégats qui forment

l'agglomération en incluant les bordures de zones non-bâties à l'intérieur des zones

urbanisées $D_{\text {bord/tot }}$;

- d'autre part, la dimension fractale de la bordure extérieure de l'agrégat principal, donc de la ville-centre $D_{\text {bord/agr }}$.

Il a été ainsi possible de calculer les indicateurs $\delta$ et $\varphi$.

49 L'agglomération berlinoise montre une dimension de surface de $D_{\text {surf }}=1,75$ qui s'approche de celle de la dimension de la bordure totale. La bordure de l'agrégat central est de $D_{\text {bord/agr }}=1,58$ et l'indice de dendricité est $d=0,89$. Il s'agit donc d'un tissu dont l'organisation spatiale s'approche de celle d'un tapis de Sierpinski. La valeur de $D_{\text {surf }}$ met en évidence la marque d'un tissu bâti très contrasté et loin d'une couverture uniforme de l'espace. La valeur basse de l'indice de fragmentation $\varphi=0,11$ montre que l'agglomération est dominée par l'agrégat principal. L'histoire de l'urbanisation de Berlin permet d'expliquer ces caractéristiques morphologiques : l'agglomération s'est étendue le long des axes du RER ( $\mathrm{S}-\mathrm{Bahn} »)$ et la politique d'aménagement tente depuis longtemps de préserver des secteurs verts qui séparent les différentes branches tentaculaires dans le but d'offrir des zones de loisir à proximité des zones habitées. Le résultat de cette politique est une forte articulation multi-échelles de zones bâties et non-bâties. 
50 Pour l'agglomération de Stuttgart, la valeur $D_{\text {surf }}$ est identique à celle de Berlin. On retrouve en effet une structure tentaculaire dans laquelle le bâti s'est développé le long de vallées bien accessibles avec la voiture et le RER. En revanche, la dimension de la bordure totale est plus élevée, tandis que celle de la bordure de l'agrégat central est légèrement plus basse par rapport à Berlin. La valeur $\varphi=0,21$ exprime ainsi une fragmentation plus forte à Stuttgart tandis que la dendricité est moins élevée $(\delta=0,78)$. En effet, la politique d'aménagement a tenté à Stuttgart de poursuivre une politique en faveur du maintien d'une bordure lisse de la zone urbanisée. En revanche, à l'intérieur de la ville existe un certain nombre d'espaces récréatifs de (parcs, squares, terrains de sports) ce qui explique la valeur légèrement plus élevée de $\varphi$.

51 Pour Londres, la situation montre une évolution bien différente. L'occupation de la surface tend vers l'uniformité $\left(D_{\text {surf }}=1,86\right)$, valeur qui est identique à celle de la bordure totale. En revanche, la bordure de l'agrégat central est $D_{\text {bor/agr }}=1,41$. Ainsi, la dendricité est moins élevée que pour les autres villes $\delta=0,68$ tandis que la fragmentation est plus forte : $\varphi=0,32$. A Londres, la politique de la préservation de la ceinture verte a empêché un étalement tentaculaire, l'agrégat central reste compact et sa bordure assez lisse.

D'autres investigations basées sur une comparaison du bâti pour plusieurs périodes ont également montré que la répartition de la surface bâtie devient en général plus uniforme dans les agglomérations suite à la motorisation. Ceci s'explique par l'amélioration progressive $\mathrm{du}$ réseau routier qui rend ainsi possible une périurbanisation quasi homogène (Frankhauser et Genre-Grandpierre (1998)). Ces résultats, obtenus pour Berlin, Munich, Strasbourg, la Ruhr et Montbéliard, ont été confirmés récemment pour Bâle (C. Tannier et D. Pumain (2004)).

\section{B. L'organisation spatiale à l'échelle des quartiers}

Pour étudier l'organisation spatiale à l'intérieur des agglomérations, une douzaine d'agglomérations européennes ont été sélectionnées et étudiées à travers des représentations cartographiques qui rendent compte de la taille réelle des bâtiments. L'échantillon comporte des agglomérations polynucléaires comme Lille ou Montbéliard, des agglomérations monocentriques comme Bâle ou Strasbourg, des espaces transfrontaliers comme Bâle, Strasbourg ou Sarrebruck et des zones métropolitaines telles que Bruxelles, Stuttgart, la Ruhr ou Milan. Un cas particulièrement intéressant est celui de la ville nouvelle de Cergy-Pontoise ${ }^{8}$.

54 Afin de définir une zone d'analyse à l'intérieur d'une agglomération, les quartiers ont été délimités dans la majeure partie des cas par une analyse radiale préliminaire qui permet d'identifier des ruptures dans l'organisation spatiale des tissus urbains (cf. figure 7).

55 L'organisation spatiale des zones sélectionnées a ensuite été étudiée en utilisant différentes méthodes d'analyse. Nous nous référons ici plus spécialement aux résultats obtenus par l'analyse de corrélation, qui s'est avérée particulièrement fiable et qui peut être utilisée à la fois pour analyser la surface bâtie et les bordures urbaines. 
Figure 7 : Le tissu bâti du centre de Stuttgart et le résultat d'une analyse radiale. On identifie deux ruptures dans la courbe du comportement scalant (à droite). Les distances indiquées en mètres correspondent à la longueur de base des carrés qui entourent le centre de comptage : ainsi les carrés répertoriés à gauche correspondent aux ruptures identifiées.
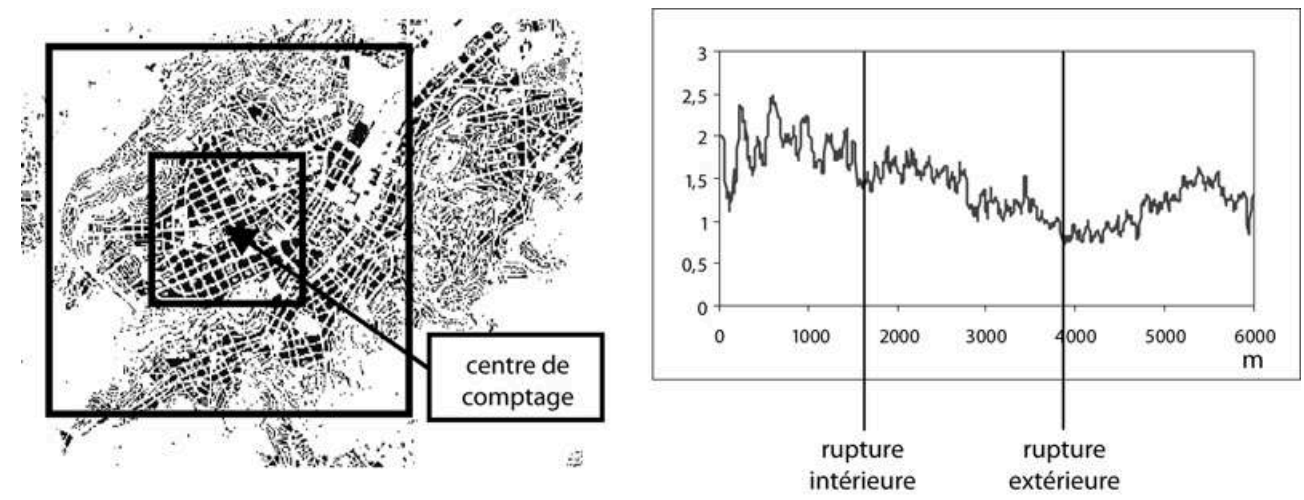

\section{L'analyse de la surface bâtie}

Pour les centres urbains, on observe pour toutes les agglomérations que la surface bâtie tend vers l'uniformité : les valeurs des dimensions fractales $D_{\text {surf }}$ varient entre 1,8 et 1,95. Des valeurs semblables sont observées pour les quartiers situés en marge de ces centres. Il s'agit de zones très denses caractérisées par une valeur foncière élevée. Dans des grandes agglomérations, constituées d'un centre principal et de centres secondaires situés dans la grande banlieue, on constate que les dimensions fractales des centres urbains des villes périphériques sont souvent moins élevées $(1,61$ à 1,81$)$. Les tissus sont donc plus contrastés. Ceci est par exemple le cas pour la région de Stuttgart : seules les villes très proches du centre principal présentent des valeurs de dimension fractale comparables à celle de Stuttgart. En revanche, dans les conurbations, comme dans le pays de Montbéliard ou dans la Ruhr, les valeurs sont comparables pour tous les noyaux urbains importants qui sont en effet tous de même taille (Montbéliard: environ $1,80$, Ruhr : 1,84 à 1,90$)$.

$\mathrm{Au}$ contraire, les dimensions fractales des quartiers périurbains varient de manière importante. Toutefois, les valeurs observées reflètent bien les différents types de tissus présents dans ces zones : l'uniformité observée pour des lotissements se situe au même niveau que celle des centre-villes (mêmes valeurs de dimension fractale). L'information transcrite par la dimension fractale est donc ici bien distincte de la notion de densité. On trouve de tels exemples en région lyonnaise: à l'Ouest de Lyon les valeurs des dimensions fractales se situent entre 1,82 et 1,99. Certains lotissements dans la périphérie de Stuttgart et de Helsinki présentent des valeurs semblables. Une telle situation reflète que la construction a été soumise à des réglementations précises (coefficient d'occupation du sol...), que les lots sont tous de même taille et que les espaces publiques ou autres équipements (écoles, commerces, équipements sportifs...) sont absents.

Des zones dans lesquelles les contraintes ont été moindres et qui montrent des tissus plus variés, sont souvent plus contrastées et leur dimension fractale est plus basse. Ainsi dans les zones périphériques de Bruxelles, Lille, Stuttgart et Bergame les valeurs 
varient entre 1,50 et 1,74. Les zones industrielles sont aussi plutôt contrastées, comme on l'observe par exemple à Lille, au Sud de Lyon et à Strasbourg.

Certains types d'habitats, conçus par des urbanistes, présentent également de forts contrastes. Ceci vaut surtout pour les cités construites dans l'esprit de la Charte d'Athènes, mais aussi les villes nouvelles (Cergy-Pontoise, Villeneuve d'Asq) dont la dimension est assez basse : elle varie entre 1,54 à 1,77. Dans ce cas l'objectif des concepteurs de créer à proximité des habitations des espaces publics à vocation différente (espaces verts de proximité, terrains de jeux, terrains de sport...) explique que leur superficie est inégale et suit un principe hiérarchique ce qui induit des valeurs de dimensions basses. De tels quartiers sont très présents en France (Montbéliard, Lyon, Strasbourg) mais existent aussi en Allemagne (Stuttgart), en revanche, ils sont absents en Belgique par exemple.

L'ensemble de ces résultats montre l'importance du contexte local de l'urbanisation sur la forme de la texture urbaine, contexte local dont l'influence paraît souvent plus importante que le cadre légal à l'échelle nationale. Ceci semble être confirmé si l'on considère les résultats obtenus pour certaines villes frontalières. A Strasbourg, des zones périurbaines montrent des valeurs semblables des deux côtés du Rhin. Dans la région de Sarrebruck, des valeurs assez basses sont observées des deux côtés de la frontière. Dans la périphérie bâloise, les différences sont un peu plus marquées : du côté suisse et du côté allemand les valeurs sont proches de 1,77, tandis que pour la partie française qui est dominée par une texture pavillonnaire les valeurs traduisent une texture plus uniforme $\left(D_{\text {surf }}=1,82\right)$.

\section{L'analyse des bordures urbaines}

61 Tandis que l'analyse de la surface bâtie se réfère directement à la surface occupée par les bâtiments, l'analyse des bordures pose des questions plus délicates. La bordure d'une ville sans remparts est un artefact : ce sont en effet les bâtiments qui délimitent à eux seuls l'espace bâti. Toutefois en considérant un tissu urbain nous avons bien l'impression de pouvoir identifier au moins approximativement une limite de la zone bâtie. Ainsi nous avons eu recourt à une méthode spécifique pour pouvoir extraire du tissu bâti une limite que l'on peut considérer, d'un point de vue morphologique, comme une approximation d'une bordure urbaine. Une justification mathématique de cette procédure a récemment été donnée dans (Frankhauser et Tannier (2005)). Cette méthode consiste à agrandir progressivement la surface des bâtiments en les entourant d'une bande de surface occupée. Au fil de telles « dilatations », des agrégats de taille de plus en plus importante se forment au détriment des vides séparant les parties occupées. En général, quelques étapes de dilatation suffisent pour faire disparaître le réseau de la voirie et les cours intérieures à l'échelle des îlots; seuls les boulevards, les couloirs traversés par les voies ferrées et les grandes places publiques subsistent alors (cf. figure $8 b$ ). Il est alors possible d'extraire la bordure de ces agrégats. Nous avons étudié la morphologie de la bordure urbaine sous deux aspects : d'une part, nous avons considéré la bordure totale de tous les agrégats comme le montre l'exemple de la figure 8c, d'autre part, nous avons étudié la bordure de l'agrégat principal (figure 8d). 
Figure 8 : La méthode d'extraction de bordure illustrée pour Valentigney (pays de Montbéliard). Une dilatation du bâti d'environ 5 à $10 \mathrm{~m}$ suffit pour faire disparaître les cours et le réseau de la voirie. Des agrégats apparaissent et il est possible d'identifier une bordure urbaine. La figure (c) montre l'ensemble des bordures tandis que, dans la figure $(d)$, seule la bordure de l'agrégat principal est extraite.
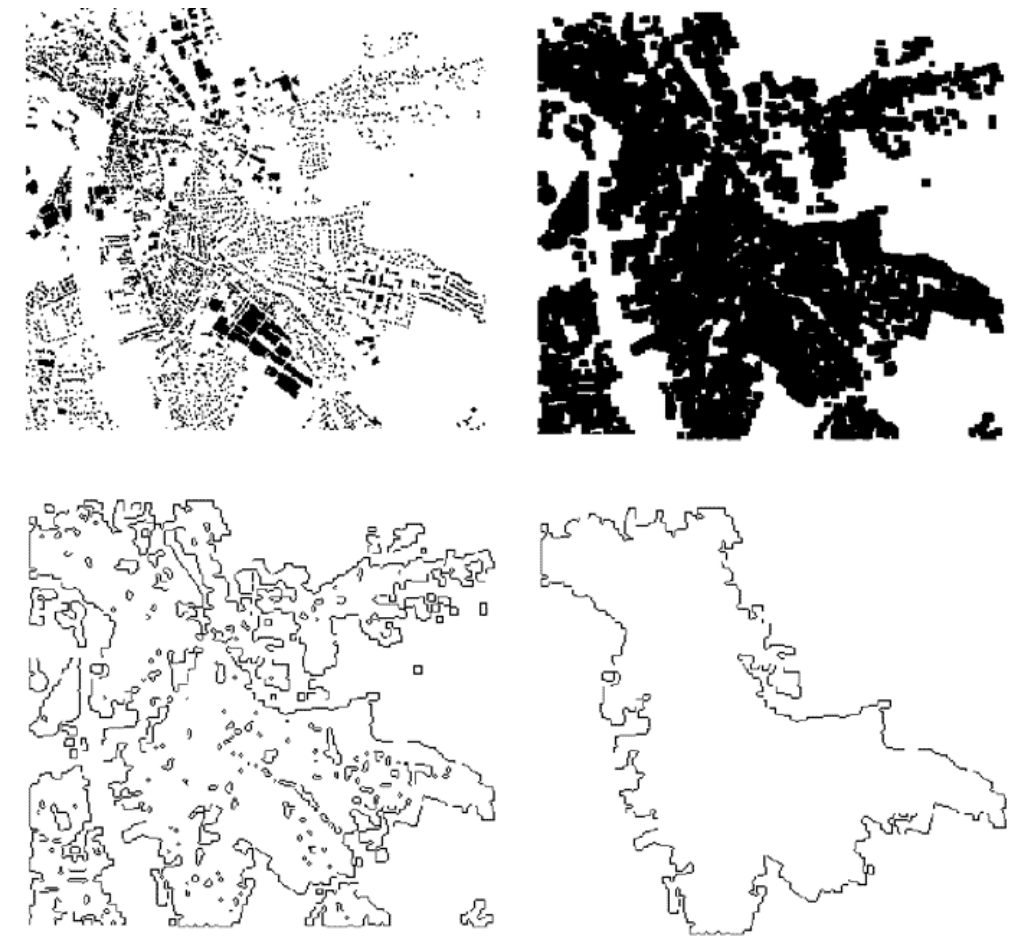

Les résultats obtenus mettent en évidence l'influence du contexte de l'urbanisation sur la morphologie des bordures. Citons d'abord quelques exemples, pour lesquels on peut supposer que le processus d'urbanisation était moins contrôlé : pour l'agglomération lilloise, on observe des dimensions fractales de la bordure qui correspondent à des tissus fragmentés et des bordures assez tortueuses. Dans la partie ouest de l'agglomération, la dimension de la bordure totale $D_{\text {bord/tot }}$ est 1,77. Elle augmente pour des distances moyennes (quartiers) à 1, 85 et elle baisse de nouveau pour des grandes distances $(1,80)$. Si l'on considère les bordures des plus grands agrégats qui se forment, on observe des valeurs $D_{\text {bord/agr }}$ qui se situent autour de 1,38 et atteignent dans un cas même 1,59! Pour les parties Est et Sud, les dimensions de la bordure des agrégats varient à peu près dans la même fourchette $(1,33$ à 1,55$)$. Dans l'agglomération lyonnaise, la situation est globalement comparable. De même, dans la périphérie de Bergame, en Italie, où l'étalement urbain n'est pas soumis à des restrictions fortes, la situation observée est similaire (les dimensions des bordures des agrégats se situent entre 1,43 et 1,46). Les valeurs les plus élevées sont celles que nous avons observé pour les zones d'urbanisation diffuse du Brabant wallon: pour Rixensart, par exemple, l'agrégat principal a une dimension de 1,66. La bordure est donc bien plus tortueuse que celle du téragone de la figure 6 dont la dimension de bordure est 1,50!

A Stuttgart la politique locale a tenté de contrôler l'étalement urbain à l'échelle du quartier, la dimension fractale de la bordure de l'agrégat principal est en effet 1,27. La politique de la ville de Fellbach, voisine de Stuttgart, n'a pas été aussi dirigiste, la bordure de l'agrégat principal est 1,45. Cependant en étudiant pour Stuttgart la 
dimension fractale de différentes parties de la bordure, on s'aperçoit que le contexte de l'urbanisation a de nouveau influencé la forme de la bordure : par exemple, on a voulu empêcher à un certain endroit que l'urbanisation empiète sur la vallée voisine, la bordure suit la crête et la dimension est $D_{\text {bord/agr }}=1,1$. En revanche dans certains quartiers périphériques où l'urbanisation s'est faite de façon plus progressive et moins contrôlée, la dimension atteint 1,70 !

Un exemple intéressant est le quartier de Botnang, ancien village situé en périphérie de Stuttgart. La politique d'aménagement a fait respecter une forme assez rectilignes pour les bordures ; la dimension de la bordure de l'agrégat principal est en effet assez basse : $D_{\text {bord/agr }}=1,19$. En revanche des îlots non-bâtis subsistent en grand nombre à l'intérieur du tissu bâti. La valeur élevée $D_{\text {bord/tot }} 1,85$ de la dimension fractale indique que ces îlots sont répartis de façon quasi uniforme dans l'espace intra-urbain.

On constate effectivement que les tissus dont la bordure est plus lisse montrent souvent une fragmentation plus importante pour l'espace intra-urbain et qu'en revanche les tissus à bordure très tortueuse sont plus compacts à l'intérieur. Ceci devient évident si on considère les deux indicateurs, l'indice de dendricité $\delta$ et l'indice de fragmentation $\varphi$ (cf. tableau 1). Rappelons que $\delta=0$ pour une figure euclidienne tandis qu'elle atteint sa valeur maximale $\delta=1$ pour le tapis de Sierpinski de la figure 2. $\varphi$ varie également entre zéro et un, la valeur minimale correspond à un objet euclidien isolé (comme un carré) et $\varphi=1$ caractérise une structure comprenant beaucoup d'agrégats de taille différente, mais dont la bordure de chacun est lisse.

Tableau 1 : Quelques indicateurs fractals pour plusieurs agglomérations : il s'agit de résultats obtenus à partir de représentations cartographiques dont les détails indiqués correspondent au moins à ceux d'une carte topographique au $1: 25.000^{9}$.

\begin{tabular}{|c|c|c|c|c|c|c|}
\hline $\begin{array}{l}\text { noyanx urbains } \\
\text { (grandes villes) }\end{array}$ & $D_{\text {surf }}$ & $\begin{array}{l}\text { villes } \\
\text { périphériques }\end{array}$ & agglomération de... & $D_{\text {surf }}$ & $d$ & $\varphi$ \\
\hline Stuttgart & 1,95 & Fellbach & Stuttgart & 1,81 & 0,75 & 0,17 \\
\hline Bâle & 1,97 & Ludwigsburg & Stuttgart & 1,73 & 0,67 & 0,35 \\
\hline Lille & 1,89 & Esslingen & Stuttgart & 1,61 & 0,87 & 0,30 \\
\hline Lyon & 1,81 & Wasquehal & Lille & 1,74 & 0,79 & 0,23 \\
\hline Bruxelles & 1,89 & Mons-en-Bareuil & Lille & 1,87 & 0,64 & 0,36 \\
\hline Essen & 1.87 & Linselles & Lille & 1,64 & 0,71 & 0,08 \\
\hline Bottrop & 1,86 & Vénissieux & Lyon & 1,79 & 0,82 & - \\
\hline Dortmund & 1,88 & St Priest & Lyon & 1,86 & 0,85 & 0,24 \\
\hline $\begin{array}{l}\text { centres d'un exemple } \\
\text { polycentrique }\end{array}$ & $D_{\text {suff }}$ & $\begin{array}{l}\text { quartiers } \\
\text { pavillonnaires }\end{array}$ & agglomération de... & $D_{\text {suaf }}$ & $\delta$ & $\varphi$ \\
\hline Montbéliard & 1,80 & Meyzieu & Lyon & 1,83 & - & - \\
\hline Valentigney & 1,87 & Lyon Ouest & Lyon & 1,82 & - & - \\
\hline Audincourt & 1,75 & Lyon Ouest & Lyon & 1,99 & - & - \\
\hline \multirow[t]{2}{*}{ Etupes } & 1.80 & Lyon Ouest & Lyon & 1,92 & - & - \\
\hline & & Benningen & Stuttgart & 1,75 & 0,54 & 0,46 \\
\hline \multirow{6}{*}{$\begin{array}{l}\text { villes noivelles } \\
\text { Cergy-Préfecture } \\
\text { Cergy/St. Christophe } \\
\text { Cergy/St. Christophe } \\
\text { Cergy/Jouy-le-Moutier } \\
\text { Villeneuve-d'Asq }\end{array}$} & $D_{\text {staff }}$ & Bissingen & Stuttgant & 1,86 & 0,53 & 0,42 \\
\hline & 1.68 & Buch & Stuttgart & 1,87 & 0,71 & - \\
\hline & 1.6 & Gablenberg & Stuttgant & 1,92 & - & - \\
\hline & 1,7 & Botnang & Stuttgart & 1,85 & 0,53 & 0,44 \\
\hline & 1,73 & & & & & \\
\hline & 1,74 & $\begin{array}{l}\text { quartiers } \\
\text { corbuséens }\end{array}$ & agglomération de... & $D_{\text {surf }}$ & & \\
\hline & & Freiberg & Stuttgart & 1,63 & & \\
\hline & & Valentigney-les Buis & Montbéliard & 1,66 & & \\
\hline & & Bethoncourt & Montbéliard & 1,70 & & \\
\hline & & Chassieu & Lyon & 1,77 & & \\
\hline
\end{tabular}



0,44 sont moyennes. Nous observons des valeurs semblables pour d'autres habitats plutôt pavillonnaires dans la région de Stuttgart pour la politique d'aménagement visaient le maintien d'une forme plus compacte. En revanche pour Fellbach, ville pour laquelle nous avons constaté que la bordure est très tortueuse, l'indice de dendricité atteint une valeur de $\delta=0,75$, mais la valeur de l'indice de fragmentation est considérablement plus basse que pour les villes citées : $\varphi=0,17$. On constate la même tendance pour Esslingen; dans les deux cas il s'agit de de villes moyennes, plus marquées par l'industrialisation.

Un exemple encore extrême est le tissu bâti de Linselle près de Lille (figure 9a). En dépit de son aspect dendritique $(\delta=0,71)$, la fragmentation est très faible $(\varphi=0,08)$. Un exemple différent est St. Priest faisant partie de l'agglomération lyonnaise (figure $9 \mathrm{~b}$ ). Dans ce cas, aussi bien la dendricité $(\delta=0,85)$ que la fragmentation $(\varphi=0,24)$ sont plus élevées qu'à Linselle, mais la fragmentation reste toutefois bien inférieure à celle de Botnang. Ainsi bien que cette ville paraisse plus «arrondie » que Linselle, son tissu est plus fragmenté à l'intérieur de la zone bâtie c'est-à-dire il contient de nombreuses zones non-bâties réparties de façon presque uniforme.

On pourrait ainsi dire que la morphologie de Linselle ressemble plutôt à celle du tapis de Sierpinski de la figure 3 pour lequel on a $\delta=1$ et $\varphi=0$, tandis que celle de St. Priest est plus proche du tapis de Sierpinski compact de la figure 4 a $(\varphi=0,89, \delta=0,11)$.

Figure 9 : Deux tissus irréguliers : Linselle en région lilloise (a) et St. Priest en région lyonnaise (b). Le tissu de Linselle est moins dendritique et moins fragmenté que celui de St. Priest.
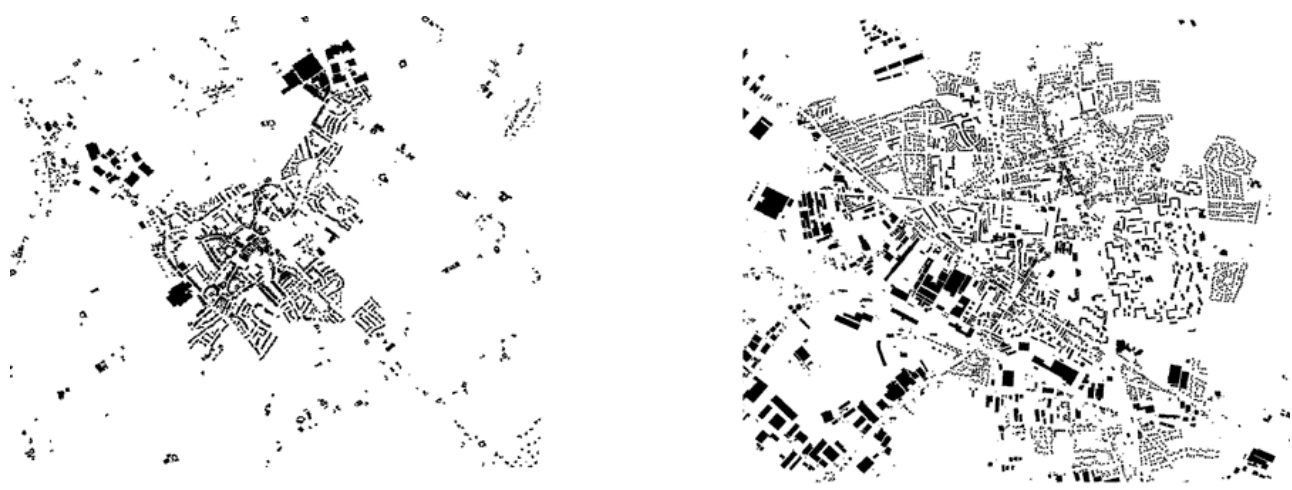

\section{E. Urbanisation et urbanisme - quelques réflexions conceptuelles}

Les résultats des analyses morphologiques ont montré que la répartition de la surface bâtie dans les tissus urbains peut être caractérisée de façon pertinente par des indicateurs fractals. Le processus d'urbanisation fait donc effectivement émerger un type d'organisation spatial particulier qui correspond au principe d'emboîtement d'échelle, caractéristique pour les objets fractals. On peut observer l'apparition progressive de ce type d'organisation spatiale en comparant le comportement fractal d'un tissu urbain à différentes dates. Dans cet objectif nous avons eu recourt à des 
analyses radiales et nous avons confronté les courbes du comportement scalant de ces analyses. On constate alors que les fluctuations autour du comportement dominant, tel qu'on les observe par exemple dans la figure 6, diminuent souvent au cours de l'urbanisation. (Frankhauser (2000)). Ceci veut dire que l'organisation spatiale de ces tissus urbains s'approche au fil du temps de plus en plus du principe d'emboitement d'échelle, caractéristique pour les fractales. En analysant la taille des tâches urbaines dans des zones métropolitaines on a pu vérifier que ce système s'approche également de plus en plus d'une organisation hiérarchique (peu de grandes tâches et un nombre croissant de tâches de plus en plus petites (Frankhauser (1992), Schweitzer et Steinbrick (2002)). Cette observation rappelle la hiérarchie des agrégats dans la fractale mixte de la figure $5 a$ et s'inscrit donc également dans une logique fractale.

Cependant si l'on considère les valeurs de la dimension fractale, on s'aperçoit que celles-ci augmentent souvent au cours du temps, c'est-à-dire qu'elles tendent vers deux. De plus en plus la surface bâtie couvre donc l'espace de façon uniforme. Ceci trouve son explication dans l'amélioration de la desserte routière des espaces ruraux situés loin des axes de transport. On touche ici au point sensible de la périurbanisation : un tel mitage urbain uniforme et omniprésent dans l'espace prériurbain augmente forcément les distances pour accéder aux centres de services et aux lieux du travail. Toutefois, les analyses ont aussi montré que certaines politiques d'aménagement produisent des tissus où les habitats se concentrent le long des axes de transport, desservis par des transport en commun et dont la dimension fractale est donc plus basse. Ceci était le cas pour Stuttgart ou Berlin.

71 Tentons maintenant de tirer quelques conclusions de ces observations au regard de l'aménagement urbain. Même si la seule analyse de la surface bâtie et des bordures urbaines ne fournit pas d'information sur l'occupation du sol des espaces interstitiels, il paraît évident qu'une répartition spatiale uniforme telle qu'on la trouve dans les centres denses ou dans certaines zones pavillonnaires n'est pas en mesure de pouvoir accueillir une grande diversité d'aménités. Ainsi, dans un lotissement, chaque propriétaire de maison bénéficie d'un jardin individuel, mais il n'existe pas d'espace public de taille suffisamment importante pour accueillir des terrains de jeux collectifs pour les enfants ou des lieux de rencontres pour les résidents. En outre, on constate souvent un manque de services banals à l'échelle du quartier. Les résidents sont obligés de se déplacer pour accéder aux divers types de services et il est évident que de tels quartiers sont des générateurs de trafic. Toutefois, si les aménités "urbaines » manquent dans les quartiers de ce genre, la densification pose aussi des problèmes car elle induit implicitement un allongement des parcours pour accéder à des zones de loisir. Ainsi, une "ville compacte» semble absurde à l'échelle d'une grande agglomération comme le montre l'exemple de la figure 10b, où les auteurs ont regroupé la surface bâtie de Berlin (figure 10a) sous forme d'un cercle. On peut supposer qu'une telle structure génèrerait des flux de trafic importants, car tous les espaces verts sont situés en bordure de l'agglomération. En outre, la bordure dont la longueur serait minimisée n'offrirait qu'à peu de résidents l'opportunité de profiter de la proximité immédiate d'un espace vert. Il est aussi évident qu'une tache urbaine compacte de cette taille ne permettrait plus une ventilation suffisante au cœur de l'agglomération.

72 L'autre tendance extrême de l'aménagement urbain serait un semis uniforme de villes de taille correspondante à celle d'une petite ville médiévale (figure 10c). Certes, un tel système fournirait à un nombre important de personnes un accès facile à une espace 
non-bâti. En revanche, on constaterait une absence totale de centralité. Tous les services «urbains» seraient dispersés dans les différentes villes. Une telle configuration spatiale obligerait également les résidents à se déplacer pour accéder aux divers services répartis dans les différents lieux. Les flux de trafic générés seraient donc très importants. De plus, le saupoudrage uniforme des habitats ne permettrait plus de disposer d'espaces naturels ou ruraux de taille conséquente.

Figure 10 : La surface bâtie de l'agglomération berlinoise (a), la même surface regroupée en disque (b) et répartie dans des petites villes à plan circulaire (surface $\left.1,67 \mathrm{~km}^{2}\right)($ selon Humpert et al. 1991).

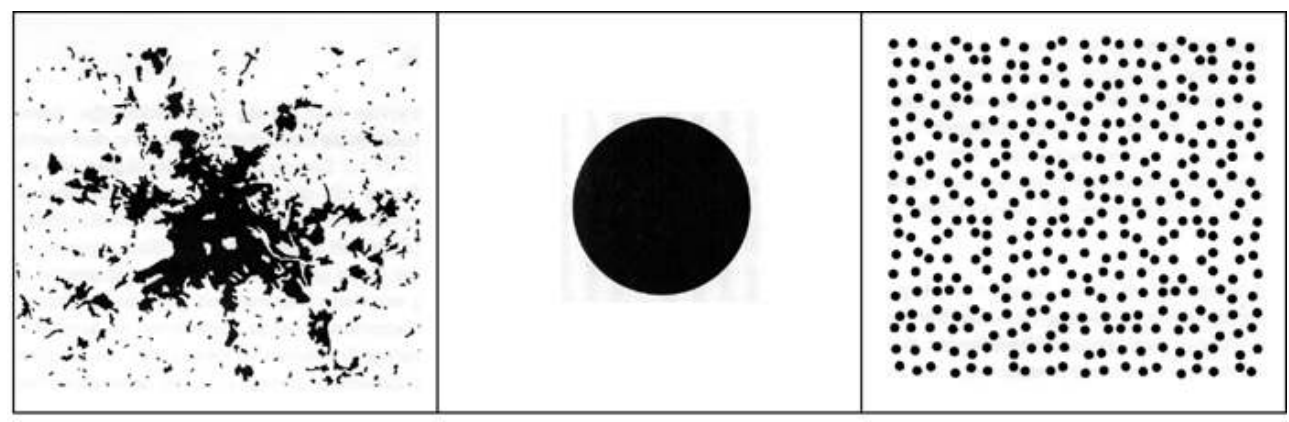

Conscients de ces contradictions, différents concepts d'urbanisme essaient de proposer des solutions en articulant les zones urbanisées et les zones rurales. Nous avons déjà cité les exemples de Berlin et Stuttgart où les aménagements tendent à concentrer l'urbanisation dans des couloirs desservis par des transports en commun. Un autre exemple serait l'agglomération de Karlsruhe: depuis la mise en place d'une système tram-train performant, les communes périphériques déclarent leurs zones constructibles à proximité des stations. Une politique de tarification conséquente et la réduction de l'offre de stationnement dans la ville-centre complètent cette stratégie. D'autres plans comme celui des doigts de gants de Copenhague s'inscrivent dans une logique similaire. (figure 11a).

Le développement axial ne garantie pas une structuration fonctionnelle à l'échelle d'une grande agglomération. Comme le fait par exemple remarquer V. Fouchier (1995), le développement de centres secondaires paraît indispensable pour réduire les flux de trafic. On retrouve un tel principe par exemple dans le concept anglo-saxon de "villages urbains ", qui tente de créer une offre de services banals à une échelle locale tout en localisant des fonctions commerciales et culturelles dans des sous-centres accessibles par des systèmes de transport à la demande. Des réseaux de transport en commun plus classiques (RER...) relient ces centres secondaires à la ville-centre qui concentrent les services rares, les centres de formation supérieure, etc. (Fouchier (1995)). Toutefois la seule existence de centres secondaires n'est pas suffisante, il paraît en effet important de réfléchir à leur localisation. Ainsi la théorie des lieux centraux de Christaller et Lösch introduit un système hiérarchique de services, mais leur implantation dans l'espace est complètement uniforme (figure 11b). Nous avons modifié ce système spatial en intercalant à différentes échelles des zones vertes entre les axes de transport. Ainsi plusieurs systèmes sont articulés : le système des espaces verts n'est pas morcelé et contient des vastes zones non urbanisées. Les zones habitées sont rapprochées des axes de transport, mais restent aussi reliés aux systèmes de zones vertes. Les services urbains sont concentrés dans les sous-centres qui peuvent être des 
points d'interconnexion des réseaux de transport en commun ou des lieux de parking relais (figure 11c).

Figure 11 : (a) Le schéma des doigts de gants pour Copenhague. (b) Le système des lieux centraux selon Christaller. (c) L'articulation multi-échelle entre zones bâties et non-bâties dans une structure fractale. (d) Une proposition d'articuler les zones bâties et le paysage ouvert dans les franges urbaines (Schöfl (1986)).
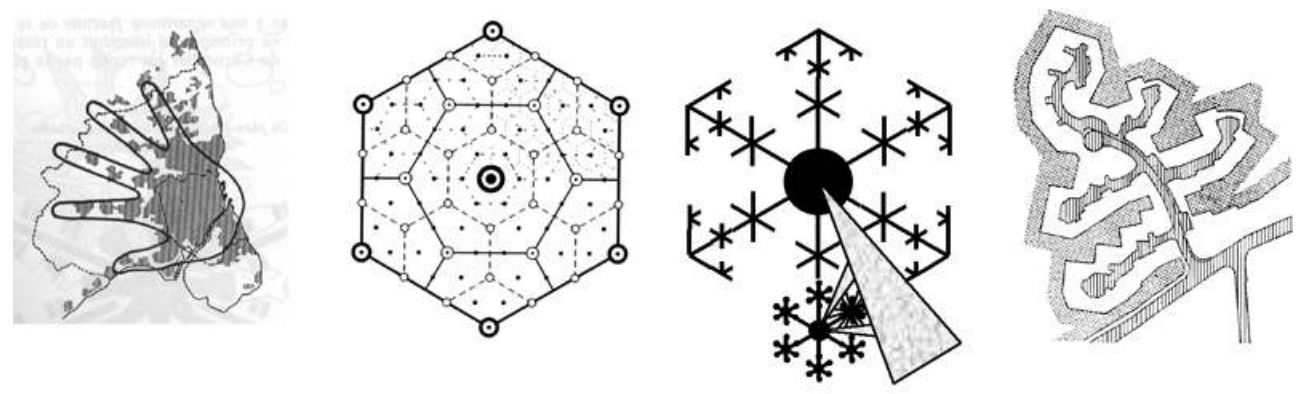

Un tel concept rappelle de nouveau certaines zones métropolitaines déjà considérées. Ainsi l'agglomération de Stuttgart compte au moins cinq centres secondaires situés à moins d'une trentaine de kilomètres de la ville-centre sur les axes principaux qui sont séparés par des zones plutôt rurales (figure 1a).

À l'échelle des quartiers les analyses de la morphologie des bordures semblent indiquer que la tentative d'imposer des bordures rectilignes n'empêche pas nécessairement l'existence d'une fragmentation à l'intérieur des tissus urbains. Ainsi on peut poser la question de savoir si une articulation entre espace urbanisé et espace vert dans les franges urbaines ne présente pas un meilleur moyen d'éviter le mitage urbain ${ }^{10}$ : le marché foncier peut alors offrir une plus grande quantité de terrains situés en frange de l'espace urbanisé, comme le montrent les deux représentations schématiques (figure $11 \mathrm{c}$ et d). Une telle ouverture visuelle au paysage semble correspondre au souhait de bon nombre de ménages qui s'installent dans le périurbain. Le principe d'emboîtement d'échelles peut servir non seulement à rapprocher "la ville et la campagne ", mais aussi à créer des proximités intra-urbaines entre des zones de plus haute densité et dans des zones pavillonnaires et ainsi, contribuer à réduire les phénomènes de ségrégation.

$\mathrm{Au}$ final, les résultats présentés mettent en évidence l'intérêt de l'approche fractale à la fois, pour concevoir des mesures morphologiques qui permettent de mieux caractériser l'organisation spatiale des tissus urbains, mais aussi comme approche conceptuelle pour réfléchir à une structuration de nouveaux espaces urbains dans l'optique d'un développement durable.

\section{BIBLIOGRAPHIE}

BATTY M., LONGLEY P. (1986) - The fractal simulation of urban structure. Environment and Planning A, 18,1143-1179. 
BATTY M., LONGLEY P. (1994) - Fractal Cities. A Geometry of Form and Function, London : Academic Press, $394 \mathrm{p}$.

BATTY M., KIM S.K. (1992) - Form Follows Function : Reformulating Urban Population Density Functions. Urban Studies, 29, 7, p. 1043-1070.

BATTY M.XIE Y. (1996) - Preliminary evidence for a theory of the fractal city, Environment and Planning A 1996, 28, 1745-1762.

BENGUIGUI L., CZAMANSKI D., MARINOV M., PORTUGALI Y. (2000) - When and where is a city fractal ? Environment and Planning B, 27 : 4, 507-519.

DE KEERSMAEKER M.-L., FRANKHAUSER P., THOMAS I. (2003) - Using Fractal Dimensions for characterizing intra-urban diversity. The example of Brussels, Geographical Analysis, vol. 35, 310-328.

DE KEERSMAEKER M.-L., FRANKHAUSER P., THOMAS I. (2004) - Dimensions fractales et réalités périurbaines, L'exemple du sud de Bruxelles. Espace géographique 3, 219-240.

DUBOIS-TAINE G., CHALAS Y. (edts.) (1997) - La ville émergente. Paris.

FOUCHIER V. (1995) - La densification : une comparaison internationale entre politiques contrastées. Les Annales de la Recherche Urbaine 67 : 94-101.

FRANÇOIS N., FRANKHAUSER P., PUMAIN D. (1995) - Villes, densité et fractalité. Les Annales de la Recherche Urbaine 67 : 54-63.

FRANK H. (1987) - Die Überwindung der Stadtbaukunst, in : Idee, Prozess, Ergebnis. Die Reparatur und Rekonstruktion der Stadt. Internationale Bauausstellung Berlin.

FRANKHAUSER P. (1988) - Fractal aspects of urban systems, in : Beiträge zum ersten Internationale Symposium des SFB 230 Teil 1, SFB 230, Stuttgart, p. 67-76.

FRANKHAUSER P. (1992) - Fractal properties of settlement structures, in : Proceedings of the 1st Internat. Semin. Struct. Morphol. Montpellier, La Grande Motte, p. 357-368.

FRANKHAUSER P. (1994) - La fractalité des structures urbaines. Paris.

FRANKHAUSER P. (1997) - L'analyse fractale un nouvel outil pour l'analyse spatiale des agglomérations urbaines, Population,vol. 52, $\mathrm{n}^{\circ}$ 4, 1005-1040.

FRANKHAUSER P. (2000) - La fragmentation des espaces urbains et périurbains - une approche fractale. In : P. H. Derycke Structures des villes, entreprise et marchés urbains. L'Harmattan, collection Emploi, Industrie et Territoire. Paris, 25-54.

FRANKHAUSER P., GENRE-GRANDPIERRE C. (1998) - La géométrie fractale, un nouvel outil d'analyse et de réflexion pour l'investigation des réseaux de transport. Cahiers Scientifiques du Transport 33, 41-78

FRANKHAUSER P. (dir.) (2003) - Morphologie des «Villes émergentes » en Europe à travers les analyses fractales, rapport de recherche, PUCA (chef du projet : G. Dubois-Taine) 242 p. http://thema.univfcomte.fr/article67.html

FRANKHAUSER P., TANNIER C. (2005) - A multi-scale morphological approach for delimiting urban areas, working-paper presented at the Conference « computers in urban planning and urban management ", London.

HUMPERT K., BRENNER K., BOHM H. (1991). - Großstädtische Agglomerationen - ein globales Problem. In : Sonderforschungsbereich 230 « Natürliche Konstruktionen » (edt) Natural structures principles, strategies and models in architecture and nature, vol. 1, Stuttgart, 39-50. 
PUMAIN D. (1981) - La dynamique des villes, Economica, Paris.

RÉMY J. (1994) - La ville : réseau alvéolaire et mobilité spatiale. In : P. PELLEGRINO (edt.) Figures architecturales, formes urbaines. Genève 113-124.

SCHÖFL G. (1986) - Minimalnetze, arcus (2), 1986.

SCHWEITZER F., STEINBRICK J. (2002) - Analysis and computer simulations of urban cluster distribution, in : Humpert et al. (eds) Fundamental Principles of Urban Growth, Wuppertal : Müller und Busmann p.142-157.

SHEN, GUOQIANG (2002) - Fractal dimension and fractal growth of urbanized areas International, Journal of Geographical Information Science, vol. 16, n 5, 419-437.

SIEVERT T. (1997) - Zwischenstadt. Bauwelt Fundamente 118 : Braunschweig, Wiesbaden, 173 p.

TANNIER C., PUMAIN D. (2004) - Fractals in urban geography : a general outline and an empirical example, Cybergeo (http://www.cybergeo.presse.fr), article accepté, à paraître.

WHITE R., ENGELEN G. (1994) - Urban systems dynamics and cellular automata : fractal structures between order and chaos. Chaos, Solitons and Fractals 4, $4: 563-583$.

Sources de données cartographiques utilisées pour les figures

Lille : base de données SIG (Communauté urbaine de Lille).

Lyon : base de données SIG (Agence d'urbanisme de Lyon) et conception ThéMa.

Montbéliard : base de données SIG (Agence d'urbanisme du CAPM) et conception ThéMa.

Stuttgart : détaillée : GIS-data (Planungsamt of Stuttgart), agrégée : conception par Städtebauliches Institut (Université de Stuttgart).

\section{NOTES}

1. Le présent texte reprend en grande partie le contenu de la présentation lors du 4e séminaire « Méthodes et approches » intitulé « Application des approches et méthodes de la géographie aux transports» organisé le 29 avril 2004 par Guillaume Faburel et Christophe Jémelin à la DRAST dans le cadre du programme PREDIT 3, dont la publication des actes est prévue.

2. Réfléchir en termes de "ville circulaire » ou de "ville compacte ", ce qui est très courant en urbanisme, signifie implicitement un travail sur des modèles de référence qui ne connaissent qu'une seule échelle. Les mesures standards destinées à quantifier l'occupation du sol s'inspirent des mêmes références : par exemple, la notion de densité renvoie à une répartition uniforme du bâti, ce qui ne correspond pas à la réalité des tissus urbains complexes et fragmentés.

3. Pour la déduction complète voir Frankhauser P. (2000).

4. Les détails sont donnés par exemple dans Mandelbrot (1983) ou Frankhauser (1994).

5. La dimension fractale décrit en effet la baisse relative de la masse bâtie en s'éloignant d'un endroit dans lequel on trouve une forte concentration de masse (une déduction est donnée dans De Keersmaecker et al. (2004)).

6. Plusieurs chercheurs ont participés à ce contrat. Les analyses ont été réalisées par D. Badariotti (Strasbourg et Saarbrücken), I. Thomas et M.-L. De Keersmaeker (Brussels), C. Tannier et B. Reitel (Basel), G. Rabino et M. Caglioni (Milan). Nous recourons plus particulièrement aux résultats obtenus à ThéMA (Besançon) par L. Quiévreux et $\mathrm{P}$. Frankhauser.

7. Ce logiciel a été développé par G. Vuidel sous la resposabilité de P. Frankhauser et $C$. Tannier dans le cadre du contrat entre le PUCA et le laboratoire ThéMA. Il est maintenant disponible sur 
le site http://fractalyse.org Vous pouvez aussi consulter le site de notre laboratoire : http://univfcomte.thema.fr, rubrique « équipes de recherche » $\rightarrow$ « ville-mobilité-territoire ».

8. Nous remercions les agences d'urbanisme de Lille et de Montbéliard, les services municipaux de Stuttgart, Bruxelles, Sarrebruck, Forbach et Kehl ainsi que l'établissement public de CergyPontoise pour avoir fourni des bases de données numériques, ainsi que le laboratoire Image et Ville (Strasbourg) pour la mise à disposition et le prétraitement de certaines bases de données. Dans d'autre cas, des bases cartographiques numérisées ont été utilisées. Les représentations cartographiques simplifiées ont été élaborées par le de l'Université de Stuttgart sous la direction de K. Humpert. Pour la présentation des résultats de recherche Frankhauser (2003).

9. Pour des quartiers situés à l'intérieur d'un bâti continu, les indices $\delta$ et $\varphi$ ne peuvent pas être calculés, car il n'existe pas de bordures.

10. On trouve ce principe d'ailleurs dans le concept de Le Corbusier ainsi que dans celui des villes nouvelles.

\section{RÉSUMÉS}

L'étalement urbain génère des tissus bâtis dont la forme paraît souvent irrégulière, sans forme apparente. Ce phénomène atteint des zones de plus en plus éloignées des centres urbains ce qui est certes discutable sous l'aspect d'un développement durable. Cependant les solutions proposées pour maîtriser l'étalement urbain recourent souvent à des modèles classiques qui s'inspirent de la ville compacte et dense. Or il est connu que ces solutions sont souvent mal accueillies par la population concernée. Nous présentons dans cet article une autre approche de la forme de ces tissus urbains qui montre que leur organisation spatiale suit, en dépit de leur aspect irrégulier, une logique particulière qui peut être décrite par la géométrie fractale. Ceci permet d'appréhender d'une autre manière les raisons socio-économiques de ce phénomène et de développer une autre approche conceptuelle de la gestion de l'étalement urbain.

Urban expansion produces an irregular urban fabric. This phenomenon influences areas which are distant from urban centres, a problem for sustainable development. Solutions proposed to reduce urban expansion are modelled on the pattern of the compact town. Generally, it is believed that these solutions are unacceptable to the people concerned. In this article we present an alternative approach to the study of urban fabric, which shows that, despite their irregular form, it is possible to describe their development using fractal analysis. This permits understanding of the socio-economic causes of this process and the development an alternative conceptual approach to the management of urban expansion.

Zeichnet man die bebaute Fläche von Städten als «Schwarzplan » so entstehen Grundrissmuster, deren Form und randliche Abgrenzung auf den ersten Blick irregulär erscheinen. Dieses Phänomen steht in starken Widerspruch zum Paradigma der "verdichteten, kompakten Stadt ", das von vielen Autoren als Paradigma nachhaltiger Stadtentwicklung betrachtet wird. Von der Stadtbevölkerung selbst werden aber solche verdichteten Strukturen weniger akzeptiert. Dort verdichten sich auch Vandalismus und andere Delikte. In diesem Artikel wird ein anderer Ansatz präsentiert, der nachweist, dass die scheinbar irregulären, offenen Grundrissmuster und Ränder von Städten einer Logik folgen, die als fraktale Geometrie beschrieben werden kann. Diese Geometrie ist nicht weniger «logisch» als die kompakte, sie hat aber anders als diese keine 
ideologischen Wurzeln, sondern folgt sozio-ökonomischen Bedingungen. Akzeptiert man diese Logik kommt man zu einer ganz anderen Bewertung der Stadtmuster.

\section{INDEX}

Schlüsselwörter : Chaostheorie, Europa, Fraktalisierung, städtische Morphologie, Suburbanisierung

Keywords : chaos theory, Europe, fractalisation, peri-urbanisation, urban morphology

Mots-clés : Europe, géométrie fractale, morphologie urbaine, périurbanisation, théorie du chaos

\section{AUTEUR}

\section{P. FRANKHAUSER}

UMR ThéMA CNRS, Université de Franche-Comté, 32 rue Mégevand, 25030 Besançon, pierre.frankhauser@univ-fcomte.fr, http://thema.univ-fcomte.fr 